\title{
Estudo dos EFeitos do Potencial Lunar em Trajetórias de Veículos EsPaCiais
}

\author{
Liana D. GonÇAlves ${ }^{1}$, Evandro M. Rocco ${ }^{1}$, RodolPho V. DE Moraes ${ }^{2}$, Antônio F. B. A. Prado ${ }^{1}$.
}

\author{
1. Instituto Nacional de Pesquisas Espaciais- INPE \\ 12227-010, São José dos Campos, SP, Brasil \\ E-mails: lianadgon@gmail.com,evandro@dem.inpe.br,prado@dem.inpe.br
}

\author{
2. Universidade Federal de São Paulo - UNIFESP \\ 12231-289, São José dos Campos, SP, Brasil \\ E-mails: rodolpho.vilhena@gmail.com
}

\begin{abstract}
Resumo — Estudos avaliando a influência do potencial gravitacional lunar, modelado por meio de harmônicos esféricos, em trajetórias de veículos espaciais são realizados de acordo com o modelo apresentado por Konopliv. Tal modelo permite considerar harmônicos esféricos até grau e ordem 100. No presente trabalho são realizados estudos analisando a influência da não homogeneidade do campo gravitacional lunar sobre a órbita de um satélite artificial nos seguintes casos: contribuição de cada termo do potencial; variação da perturbação em função da inclinação da órbita; atuação do sistema de controle, a fim de minimizar os efeitos perturbativos sobre a órbita de um satélite artificial. As manobras de correção orbitais de satélites lunares são simuladas utilizando empuxo contínuo e controle de trajetória em malha fechada.
\end{abstract}

Palavras-chave — Potencial gravitacional, astrodinâmica, satélites artificiais, movimento orbital, correção orbital

\section{Introdução}

Se ignorarmos a existência de forças perturbadoras, o movimento orbital dos satélites artificiais é uma cônica em um plano fixo, com tamanho e excentricidade constantes. Porém, a existência de tais forças tende a causar variações nos elementos que caracterizam a órbita do satélite artificial, fazendo com que a trajetória do satélite varie com o tempo.

Para estudar as perturbações devido a não esfericidade do campo gravitacional lunar é usado o modelo LP100K, com o objetivo de fazer uma análise da influência do grau e ordem dos harmônicos gravitacionais na órbita de um satélite artificial lunar. Tais efeitos perturbadores são inseridos no simulador de trajetória Spacecraft Trajectory SimulatorSTRS, Rocco (2008), para que seja feito o controle orbital com o objetivo de minimizar ao máximo os desvios e erros de regime, fazendo uso de propulsão contínua e de controle de trajetória orbital em malha fechada. Uma descrição mais detalhada do controle de trajetória orbital pode ser encontrado em Rocco (2012). Estudos e análises de tal influência, bem como simulações considerando a perturbação devido ao potencial gravitacional lunar sobre a órbita de um satélite artificial, podem ser encontrados em Gonçalves (2013).

\section{Potencial Gravitacional Lunar}

O potencial gravitacional da Lua é expresso pela expansão dos coeficientes harmônicos esféricos normalizados, dado pela Equação (1) (Konopliv, 2001; Kuga, 2011):

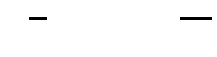

em que: $n$ é o grau e é a ordem, é a constante gravitacional e é o raio lunar equatorial. são os polinômios associados de Legendre normalizados. é o raio vetor (distância), $\emptyset$ é a latitude e $\lambda$ é a longitude.

\section{O Modelo LP100K}

O campo gravitacional lunar foi até então determinado por meio de dados de missões lunares já realizadas. Uma das missões de maior contribuição foi a Lunar Prospector, (19981999), terceira missão do programa de exploração da NASA, denominado Discovery, que forneceu a primeira medição do campo gravitacional lunar. Visto que, até o momento da modelagem utilizando os dados da missão LP, não houve observação direta do lado oculto da Lua, as informações a respeito da gravidade vem do efeito em longo prazo observado na órbita dos satélites.

O modelo apresentado por Konopliv, denominado é uma representação dos harmônicos esféricos devido à gravidade planetária, baseada no potencial gravitacional do corpo celeste, dado pela Equação (1). 
A saída calculada pelo modelo fornece as componentes $x, y$ e $z$ para a aceleração da gravidade em cada instante de tempo ao longo da órbita de um satélite artificial, sendo possível considerar os harmônicos esféricos até grau e ordem 100. Por meio de uma comparação feita entre a aceleração da gravidade de um campo central e a aceleração da gravidade fornecida pelo modelo de Konopliv, é obtido o incremento da velocidade que o termo perturbador aplica ao veículo espacial, possibilitando, por meio do problema inverso, a obtenção dos elementos keplerianos que caracterizam a órbita do satélite artificial, afim de que seja feita uma análise do movimento orbital.

\section{Simulações e Resultados}

Visto que o objetivo do trabalho é analisar a influência do potencial gravitacional lunar na órbita de um satélite artificial, foram realizados três estudos, descritos a seguir.

Primeiramente fazemos um estudo visando analisar a influência de cada termo do potencial gravitacional na órbita de um satélite artificial. Essa tarefa foi feita de uma forma indireta, obtendo-se os valores dos incrementos de velocidade aplicados por $N$ termos do potencial durante uma órbita do veículo espacial e a seguir repete-se esse cálculo para $N+1$ termos. Fazendo a diferença entre os incrementos de velocidade, obtém-se a contribuição da adição de mais termos no potencial.

Esse estudo é então feito em função da variação da inclinação, com o objetivo de mostrar a influencia desse elemento orbital na perturbação sentida pelo veículo espacial.

E, por fim, a partir da análise feita anteriormente com relação à variação da inclinação, para os casos extremos, em que existe maior e menor influência do potencial gravitacional lunar, é analisada a força perturbativa aplicada sobre o satélite e o empuxo necessário para a realização de manobras de correção orbital, a fim de que sejam minimizados os efeitos perturbativos sobre o satélite artificial nos casos extremos, em que se deseja manter a órbita kepleriana o tempo todo.

A situação em que a variação dos elementos orbitais é usada com o objetivo de minimizar o consumo de combustível para manutenção orbital é usual na prática. Porém, o objetivo aqui é medir o efeito da adição de cada termo do potencial e não encontrar a estratégia que minimize o consumo de combustível. Uma ideia similar foi feita em Prado (2013) para um veículo espacial que viaja em torno da Terra, perturbado pela Lua e pelo Sol.

\subsection{Incremento de Velocidade}

Vamos iniciar nosso estudo pela análise da importância de cada termo do potencial gravitacional lunar.

Essa tarefa é repetida 100 vezes, cada vez adicionando-se o próximo termo do potencial gravitacional, ou seja, foram feitas, para cada termo do potencial gravitacional lunar, uma simulação durante o período de uma órbita. Para todas as simulações não foram realizadas manobras de correção ou transferência, visto que objetiva-se analisar os efeitos da perturbação devido ao potencial gravitacional na órbita de um satélite artificial.

Dessa forma é obtida a variação de velocidade total aplicada sobre o satélite (integral no tempo da aceleração causada pelos termos perturbadores), que tem seu movimento governado apenas pelo potencial da Lua de acordo com o modelo de Konopliv. Essa medida calcula o efeito de adicionarmos um novo elemento ao potencial gravitacional da Lua, e não a contribuição de cada termo individualmente. Após essas simulações é feita a subtração da contribuição dos termos anteriores, mostrando assim o quanto a adição de um novo termo altera as características orbitais.

A Figura 1 mostra a variação total da velocidade decorrente da não homogeneidade da distribuição de massa lunar, com a dinâmica que leva em conta $N+1$ termos do potencial gravitacional menos a mesma variação levando em conta $\mathrm{N}$ termos do potencial gravitacional.

As condições iniciais da órbita são: semieixo: maior $1900000 \mathrm{~m}$; excentricidade: 0,001; inclinação: $45^{\circ}$; ascensão reta do nodo ascendente: $20^{\circ}$; argumento do periapside: $100^{\circ}$; anomalia média: $1^{\circ}$.

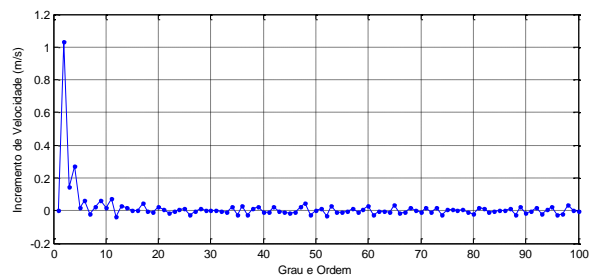

Figura 1- Diferença entre a variação de velocidade aplicada ao veículo espacial com $N+1$ e $\operatorname{com} N$ termos do potencial lunar.

Nota-se o esperado domínio do primeiro termo do potencial perturbador como observado em Carvalho (2008), Carvalho (2009a), Carvalho (2009b) e Carvalho (2010). Porém, também é possível perceber que os resultados dos próximos termos não são monotonicamente decrescentes, o que significa que um termo pode ser de ordem mais elevada e ainda assim fornecer uma contribuição relevante para o 
incremento de velocidade do satélite como estudado por Gonçalves (2013). Um estudo semelhante pode ser encontrado em Ramanan (2005).

Termos negativos aparecem na Figura 1, o que significa que a adição deste termo no potencial gravitacional lunar diminui a perturbação sofrida pelo veículo espacial, reduzindo assim a variação de velocidade requerida para manutenção da órbita kepleriana. Em outras palavras, este termo ajuda a controlar o satélite artificial, pois o seu efeito é contrário a resultante dos termos anteriores, logo a favor do sistema de controle.

\subsection{Inclinação}

Como visto no item 4.1, cada termo do potencial gravitacional lunar exerce uma influência diferente na órbita de um satélite artificial.

Nosso objetivo agora é analisar o efeito da inclinação do satélite artificial lunar na perturbação de sua órbita. Um estudo de um satélite artificial perturbado pelo potencial gravitacional para o caso da inclinação crítica é realizado em Carvalho (2009a) e Carvalho (2010).

Para isso, foram obtidos, por meio do modelo apresentado por Konopliv, os valores do incremento de velocidade sobre um satélite artificial lunar em função da inclinação. Para evitar um excesso de resultados, baseados na Figura 1, que mostra a estabilidade atingida após os primeiros termos do potencial gravitacional lunar, as simulações foram efetuadas considerando até grau e ordem 2, 10, 50 e 100 . Os resultados estão mostrados na Figura 2

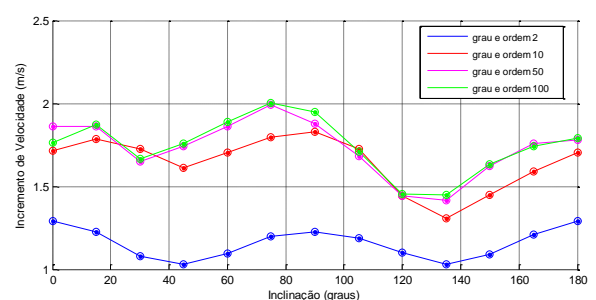

Figura 2 - Variação de velocidade fornecida pela perturbação como função da inclinação da órbita do veículo espacial e do número de termos considerados para o potencial lunar.

Foram analisadas órbitas com os seguintes valores de inclinação: $0^{\circ}, 15^{\circ}, 30^{\circ}, 45^{\circ}, 60^{\circ}, 75^{\circ}$, $90^{\circ}, 105^{\circ}, 120^{\circ}, 135^{\circ}, 150^{\circ}, 165^{\circ}$ e $180^{\circ}$, todos com as seguintes condições iniciais: semi-eixo: maior $1900000 \mathrm{~m}$; excentricidade: 0,001; ascensão reta do nodo ascendente: $20^{\circ}$; argumento do periapside: $100^{\circ}$; anomalia média: $1^{\circ}$.
Podemos perceber uma diferença significativa quando considerarmos apenas grau e ordem 2 e quando são considerados todos os termos até grau e ordem 10, 50 e 100. Também é notado que existem valores de inclinação aonde a perturbação gerada pelo potencial gravitacional lunar é maior. Analisando o caso de maior precisão do modelo de Konopliv (grau e ordem 100), podemos concluir que o valor de inclinação de $75^{\circ}$ apresenta maior valor de incremento de velocidade, e o valor de inclinação de $135^{\circ}$ apresenta o menor valor de incremento de velocidade, sendo a diferença entre eles de aproximadamente $0,55 \mathrm{~m} / \mathrm{s}$, que é bastante significativa na escala de valores obtidos.

Nota-se também que as simulações realizadas com 50 e 100 termos do potencial são muito similares entre si, enfatizando assim a pequena contribuição dos termos entre ordem 50 e 100, como esperado. Mesmo a caso considerando apenas 10 termos já é bastante similar aos casos com 50 e 100 termos, demonstrando assim o forte domínio dos primeiros termos. Porém existem diferenças significativas entre considerar apenas o primeiro termo não kepleriano e os demais até o número de 10 .

\subsection{Manobras de correção orbital}

Com o objetivo de minimizar os efeitos perturbativos devido à distribuição não uniforme de massa da Lua, são realizadas duas simulações para os casos de maior e menor influência do potencial gravitacional lunar sobre a órbita de um satélite artificial em função da inclinação.

Como visto no item 4.2, os valores extremos são $75^{\circ}$ e $135^{\circ}$, sendo, para ambos os casos, analisados a força devido a não uniformidade do campo gravitacional lunar, o empuxo e a comparação entre a perturbação, a atuação do sistema de controle que objetiva minimizar ao máximo os efeitos perturbativos sobre o veículo espacial e o comportamento da inclinação para os casos de $75^{\circ}$ e $135^{\circ}$, para as situações em que o sistema de controle não é acionado e em que o sistema de controle atua no sentido de atenuar ao máximo os efeitos perturbativos.

As Figuras 3 e 5 mostram a força perturbativa sobre o satélite, ou seja, a força que a perturbação exerce sobre o veículo, que acaba por tirá-lo da trajetória kepleriana. As Figuras 4 e 6 mostram o empuxo aplicado sobre o satélite com o objetivo de corrigir os efeitos da força perturbativa e mantê-lo na trajetória kepleriana, para os casos de inclinação $75^{\circ}$ e $135^{\circ}$, respectivamente. 


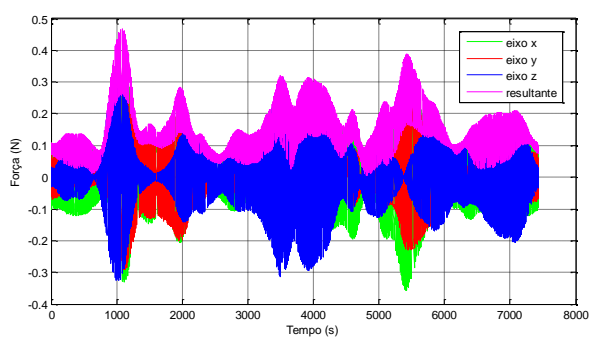

Figura 3 - Força aplicada sobre o satélite a cada instante de tempo para o caso em que a inclinação é de $75^{\circ}$.

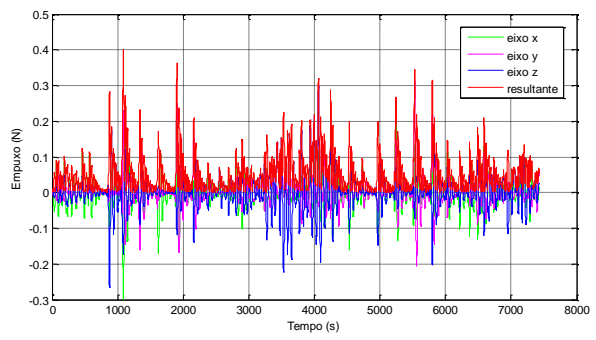

Figura 4 - Empuxo aplicado ao satélite a cada instante de tempo para o caso em que a inclinação é de $75^{\circ}$.

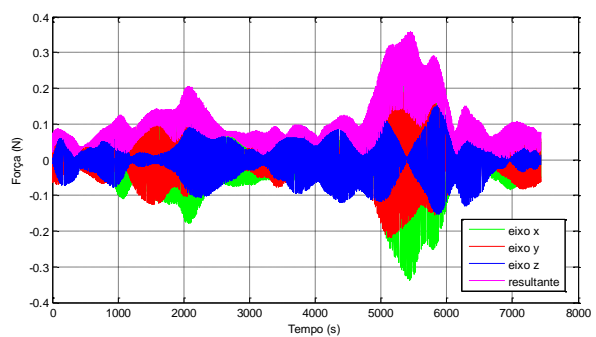

Figura 5 - Força aplicada sobre o satélite a cada instante de tempo para o caso em que a inclinação é de $135^{\circ}$.

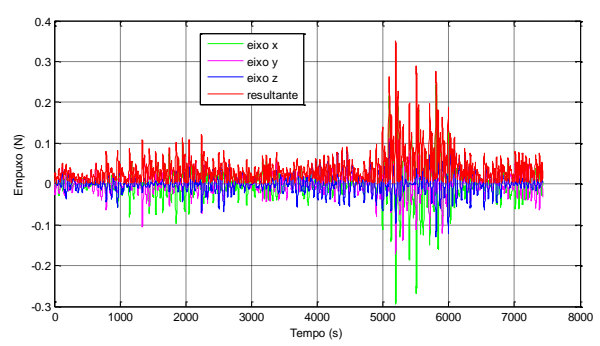

Figura 6 - Empuxo aplicado ao satélite a cada instante de tempo para o caso em que a inclinação é de $135^{\circ}$.

Pelas Figuras 3 a 6 podemos perceber que o empuxo é aplicado com o objetivo de corrigir a perturbação sobre $o$ satélite. Os picos existentes nas Figuras 4 e 6 aparecem devido à maior intensidade da força perturbativa sobre o satélite.

As Figuras 3 e 5, mostram que o potencial gravitacional lunar apresenta um comportamento bastante inconstante, dificultando a atuação do sistema de controle.

Tal situação é mostrada nas Figuras 7 a 12, que apresentam a perturbação sobre o satélite, a atuação do sistema de controle e a resultante entre a perturbação e sistema de controle para o caso de inclinação $75^{\circ}$ e $135^{\circ}$, respectivamente.

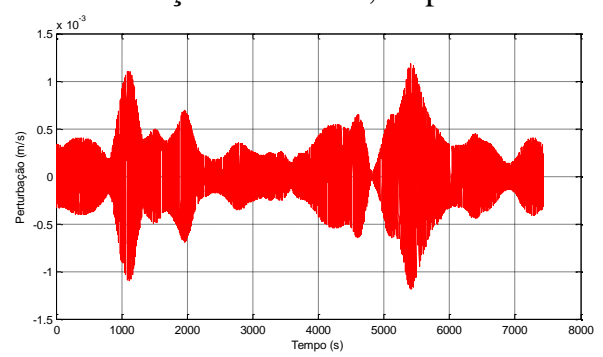

Figura 7 - Perturbação sobre o satélite para o caso de inclinação $75^{\circ}$.

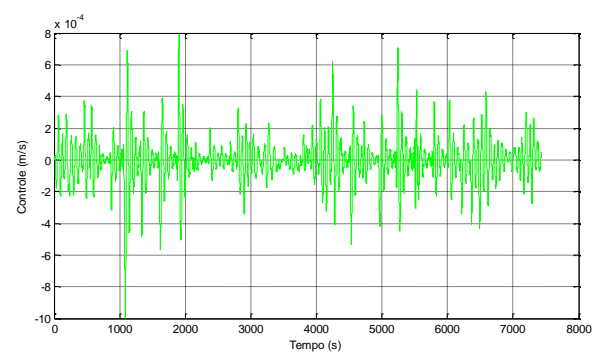

Figura 8 - Controle sobre o satélite para o caso de inclinação $75^{\circ}$.

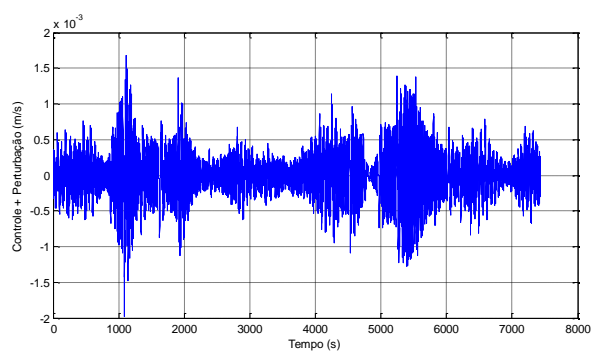

Figura 9 - Força resultante sobre o satélite para o caso de inclinação $75^{\circ}$.

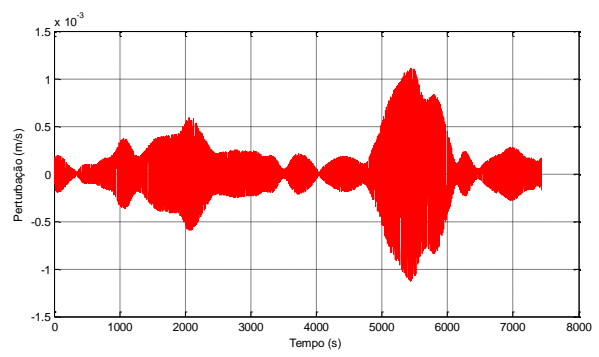

Figura 10 - Perturbação sobre o satélite para o caso de inclinação $135^{\circ}$. 


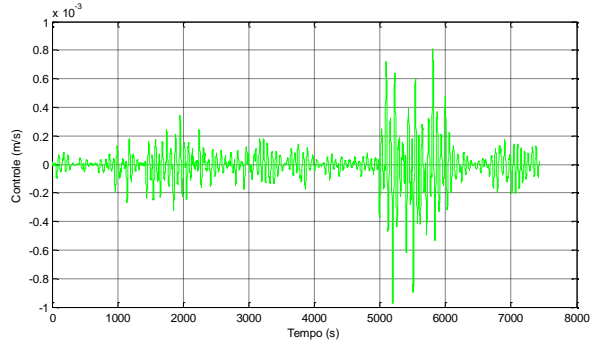

Figura 11 - Controle sobre o satélite para o caso de inclinação $135^{\circ}$.

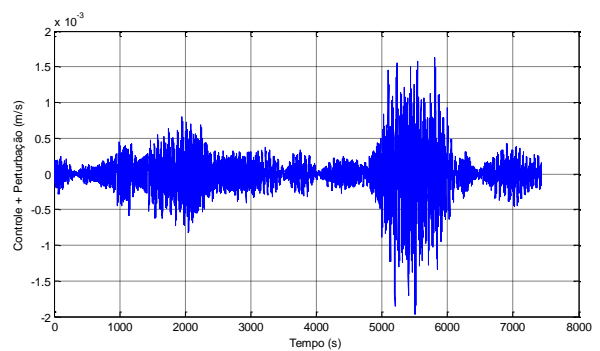

Figura 12 - Força resultante sobre o satélite para o caso de inclinação $135^{\circ}$.

Pelas Figuras 7 a 12 podemos ver que o sistema de controle é capaz de atuar no sentido de minimizar os efeitos perturbativos sobre a órbita do satélite artificial lunar, fazendo com que o satélite mantenha-se o mais próximo possível da trajetória kepleriana.

As Figuras 13 a 16 mostram o comportamento das inclinações de valores $75^{\circ} \mathrm{e}$ $135^{\circ}$, com e sem o acionamento do sistema de controle, que atua a fim de minimizar os efeitos da perturbação devido a não uniformidade do potencial lunar.

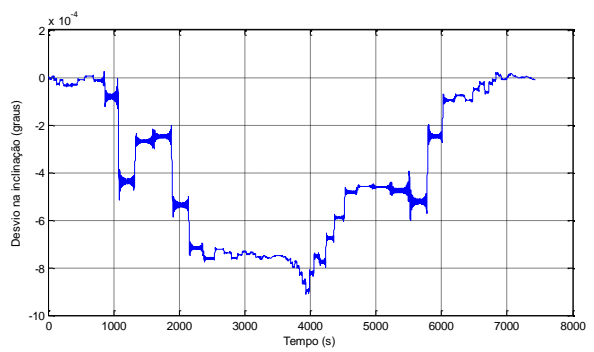

Figura 13 - Desvio na inclinação para o caso de inclinação $75^{\circ}$ sem manobras de correção orbital.

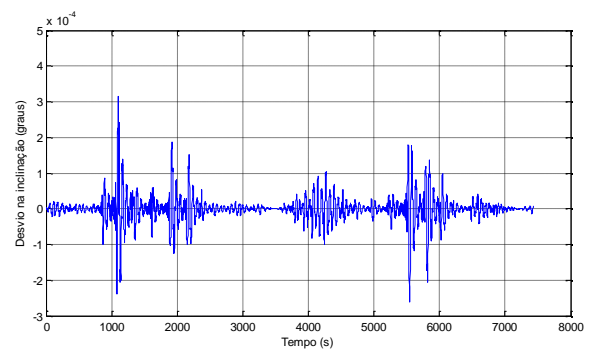

Figura 14 - Desvio na inclinação para o caso de inclinação $75^{\circ}$ com manobras de correção orbital.

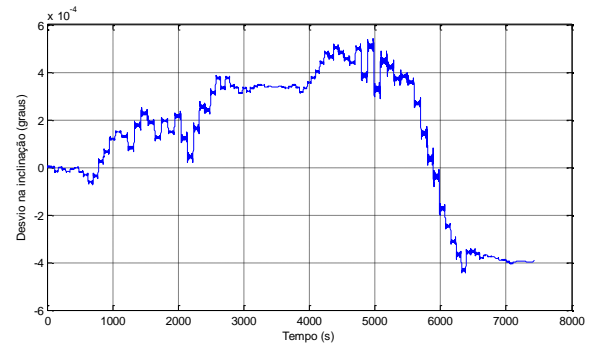

Figura 15 - Desvio na inclinação para o caso de inclinação $135^{\circ}$ sem manobras de correção orbital.

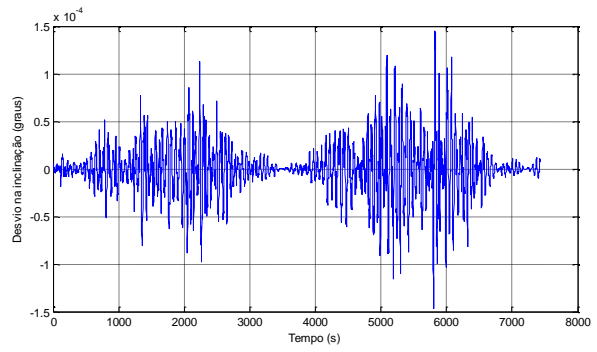

Figura 16 - Desvio na inclinação para o caso de inclinação $135^{\circ}$ com manobras de correção orbital.

O resultado obtido na Figura 13, que apresenta o caso de inclinação $75^{\circ}$ sem manobras de correção orbital, mostra que com a ausência do sistema de controle o desvio da inclinação pode chegar a $-8 \times 10^{-4}$ graus. Porém, o resultado obtido na Figura 14 mostra que com a atuação do sistema de controle o desvio da inclinação não chega a $0,5 \times 10^{-4}$ graus, com alguns picos que a, no máximo, $3 \times 10^{-4}$ graus.

Para o caso em que a inclinação é de $135^{\circ}$, Figuras 15 e 16, vemos que o desvio chega num desvio máximo de $-4 \times 10^{-4}$ graus. Tal variação é menor do que a encontrada para o caso de inclinação $75^{\circ}$, pois esta inclinação é menos perturbada. Com a presença do sistema de controle, o desvio da inclinação permaneceu em torno de $0,5 \times 10^{-4}$ graus, com alguns picos que podem chegar a $1 \times 10^{-4}$ graus.

\section{Conclusões}

Os resultados apresentados neste trabalho estão de acordo com o modelo do potencial lunar desenvolvido por Konopliv (2001), que mostra a correlação entre a aceleração da gravidade lunar e a topografia, evidenciando a flutuação da aceleração da gravidade devido a distribuição não uniforme de massa lunar.

$\mathrm{O}$ estudo feito para analisar o efeito de cada termo do potencial gravitacional sobre a órbita de um satélite artificial lunar mostrou que o primeiro termo (grau e ordem 2) domina o movimento, mas as contribuições dos demais termos são relevantes, principalmente dos 10 primeiros termos. Os demais são responsáveis por contribuição bem menores. 
Valores negativos aparecem neste estudo, evidenciando que alguns termos atuam no sentido contrario à resultante anterior, ajudando assim a manter a órbita do satélite artificial próximo à órbita kepleriana.

Os valores de inclinação estudados mostraram que a variação da inclinação pode alterar a perturbação sofrida pelo satélite artificial significativamente.

Os estudos mostram que a perturbação sobre um satélite artificial devido à distribuição não uniforme de massa da Lua é consideravelmente inconstante, o que exige atuação intensa do sistema de controle para atenuar os desvios causados na trajetória.

Podemos observar que os desvios nas variáveis de estado foram sempre valores pequenos, ou seja, o sistema de controle foi capaz de reduzir o erro nas variáveis de estado por meio da atuação dos propulsores.

\section{Referências Bibliográficas}

Carvalho, J. P. S.; Moraes, R. V; Prado, A. F. B. A. Semi-analytic theory of a moon artificial satellite considering lunar oblateness and perturbations due to a thirdbody in elliptic orbit. In: Brazilian Conference on Dynamics Control, and Aplications, 7, 2008, Presidente Prudente. Proceedings...Presidente Prudente, 2008.

Carvalho, J. P. S. Moraes, R. V; Prado, A. F. B. A. Non-sphericity of the moon and critical inclination. In: Congresso Nacional de Matemática Aplicada e Computacional, 32, 2009, Cuiabá, Brasil.Proceedings...Cuiabá, 2009(a).

Carvalho, J. P. S. Moraes, R. V.; Prado, A. F. B. A. Non-sphericity of the Moon and near Sun-synchronous polar lunar orbits, Mathematical Problems in Engineering Article ID 740460, 24 pages. DOI: $10.1155 / 2009 / 740460$

Carvalho, J. P. S. Moraes, R. V.; Prado, A. F. B. A. Some orbital characteristics of lunar artificial satellites, Celestial Mechanics \& Dynamical Astronomy, v. 108, n. 4, p. 371-388, DEC 2010. DOI:

10.1007/s10569-010-9310-6

Gonçalves, L. D. Manobras Orbitais de Satélites Artificiais Lunares com Aplicação de Propulsão Contínua. 2013. Dissertação (Mestrado em Mecânica Espacial e Controle) - Instituto Nacional de Pesquisas Espaciais (INPE), São José dos Campos. 2013.

Konopliv, A. S.; Asmar, S. W.; Carranza, E.; Sjogren, W. L.; Yuan, D. N. Recent gravity models as a result of the lunar prospector mission, Icarus, Vol. 150, pp. 118, Academic Press, 2001.

Kuga, H.K.; Carrara, V.; Kondapalli R. R. Satélites Artificiais - Movimento Orbital. INPE - São José dos Campos, 2011. 111 p. Disponível em: <http://urlib.net/8JMKD3MGP7W/3ARJ3 $\mathrm{NH}>$.

Rocco, E. M. Perturbed orbital motion with a PID control system for thetrajectory. In: Colóquio Brasileiro de Dinâmica Orbital, 14, Águas de Lindóia,2008. Resumos...2008.

Rocco, E. M. Controle de trajetória com propulsão contínua para missões do tipo drag-free. In: Congresso Nacional de Engenharia Mecânica, 7, 2012, São Luís, Brasil.Proceedings...São Luís, 2012.

Prado, A.F.B.A., "Searching for Orbits with the Minimum Fuel Consumption for StationKeeping Maneuvers: Application to LuniSolar Perturbations." Mathematical Problems in Engineering (Print), Volume 2013(2013), Article ID 415015, 11 pages.

Ramanan, R. V.; Adimurthy, V. An analysis of near-circular lunar mapping orbits. J. Earth Syst. Sci. 114, No. 6, December 2005, pp. 619-626. DOI: 10.1007/BF02715946 